\title{
HYPONORMAL TOEPLITZ OPERATORS AND EXTREMAL PROBLEMS OF HARDY SPACES
}

\author{
TAKAHIKO NAKAZI AND KATSUTOSHI TAKAHASHI
}

Dedicated to Professor Tsuyoshi Ando on his sixtieth birthday

\begin{abstract}
The symbols of hyponormal Toeplitz operators are completely described and those are also studied, being related with the extremal problems of Hardy spaces. Moreover, we discuss Halmos's question about a subnormal Toeplitz operator when the self-commutator is finite rank.
\end{abstract}

\section{INTRODUCTION}

Let $L^{p}$ be the Lebesgue space on the unit circle $\Gamma$ and let $H^{p}$ be the corresponding Hardy space for $1 \leq p \leq \infty$. The Toeplitz operator $T_{\phi}$ with symbol $\phi$ in $L^{\infty}$ is the operator on $H^{2}$ defined by $T_{\phi} x=P(\phi x)$ for $x$ in $H^{2}$, where $P$ is the orthogonal projection of $L^{2}$ onto $H^{2}$. Brown and Halmos [3] began the systematic study of the algebraic properties of Toeplitz operators and showed that $T_{\phi}$ is normal if and only if $\phi=\alpha u+\beta$ where $\alpha$ and $\beta$ are complex numbers and $u$ is a real-valued function in $L^{\infty}$. A characterization of symbols of hyponormal Toeplitz operators is known [5]. An operator $A$ is called hyponormal if its self-commutator $\left[A^{*}, A\right]=A^{*} A-A A^{*}$ is positive. However the exact descriptions of symbols of hyponormal Toeplitz operators are not known. The main purpose of this paper is to give such descriptions as those of Brown and Halmos for normal Toeplitz operators. The symbol of a hyponormal Toeplitz operator $T_{\phi}$ satisfies that $\phi-g=k \bar{\phi}, g \in H^{\infty}$, and $k \in H^{\infty}$ with $\|k\|_{\infty} \leq 1$ (see Proposition 1). Therefore $|\phi-g| \leq|\phi|$ a.e. on $\Gamma$, and hence our study is related with the extremal problems of Hardy spaces $H^{\infty}$ and $H^{1}$. In his paper "Ten problems in Hilbert space" [9], Halmos raised the question: "Is every subnormal Toeplitz operator either normal or analytic?" A Toeplitz operator $T_{\phi}$ is analytic if its symbol $\phi$ is in $H^{\infty}$. Cowen and Long [6] answered negatively Halmos's question. Abrahamse [1] gave a very general sufficient condition for the answer to be yes, after previous works of [10] and [2]. In this paper we give a simple proof of Abrahamse's theorem and a new sufficient condition for the answer to be yes. A subnormal operator is always

Received by the editors May 7, 1991.

1991 Mathematics Subject Classification. Primary 47B35, 47B20; Secondary 30D55.

Key words and phrases. Hyponormal operator, subnormal operator, Toeplitz operator, Hardy space, extremal problem.

This research was partially supported by Grant-in-Aid for Scientific Research, Ministry of Education. 
hyponormal. Hence it is desirable to describe symbols of subnormal Toeplitz operators in general.

The paper is arranged as follows. In $\S 2$, we give the general descriptions of symbols of hyponormal Toeplitz operators. In $\S \S 3$ and 4 , we study the relation between the study of the symbols of hyponormal Toeplitz operators and the extremal problems of Hardy spaces. In $\S 5$, we study Toeplitz operators of symbols of bounded type. In $\S \S 6$ and 7, the self-commutators of hyponormal Toeplitz operators are studied. In $\S 8$, we discuss a positive answer for Halmos's question.

Throughout this paper we use the following lemma which was essentially proved by Cowen [5].

Lemma 1. $T_{\phi}$ is hyponormal if and only if there exist two functions $k$ and $g$ in $H^{\infty}$ such that $\phi=k \bar{\phi}+g$ and $\|k\|_{\infty} \leq 1$.

Proof. Let $\phi \in L^{\infty}$ and $\phi=h+\bar{l}$ where $h$ and $l$ are in $H^{2}$. Cowen [5, Theorem 1] showed that $T_{\phi}$ is hyponormal if and only if $l=c+T_{\bar{k}} h$ for some constant $c$ and some function $k$ in $H^{\infty}$ with $\|k\|_{\infty} \leq 1$. If $\phi=k \bar{\phi}+g$, $g \in H^{\infty}$, and $k \in H^{\infty}$ with $\|k\|_{\infty} \leq 1$, then

$$
\phi-k \bar{\phi}=\bar{l}-k \bar{h}+h-k l \text {. }
$$

Therefore $\bar{l}-k \bar{h} \in H^{2}$ and hence $l=c+T_{\bar{k}} h$ for some constant $c$. Thus $T_{\phi}$ is hyponormal. For the converse the proof above is reversible.

\section{GeNeral DESCRIPTION OF SYMBols}

In this section, the general descriptions of symbols of hyponormal Toeplitz operators are given. Two kinds of symbols of Toeplitz operators in Propositions 1 and 2 are typical and the arbitrary symbol is a combination of these two symbols. If $q$ is an inner function then $q=(1+q)^{2} /|1+q|^{2}$. We define $q^{1 / 2}$ as in the following:

$$
q^{1 / 2}=(1+q) /|1+q| \text {. }
$$

For an arbitrary nonzero function $k$ in $H^{\infty}$, if $q$ is the inner part and $h$ is the outer part then put $k^{1 / 2}=q^{1 / 2} h^{1 / 2}$. We should note that $\left(z^{2}\right)^{1 / 2} \neq z$. For a measurable set $E$ of $\Gamma, \chi_{E}$ denotes the characteristic function of $E$.

Proposition 1. If $\phi=q^{1 / 2} u+g$ where $q$ is inner, $u$ is a real function in $L^{\infty}$, and $g$ is a function in $H^{\infty}$ with $q \bar{g}$ in $H^{\infty}$, then $T_{\phi}$ is hyponormal.

Proof. Since $\phi-q \bar{\phi}=g-q \bar{g}$ and $q \bar{g} \in H^{\infty}, \phi-q \bar{\phi}$ belongs to $H^{\infty}$. Lemma 1 implies that $T_{\phi}$ is hyponormal.

Proposition 2. If $\phi=(k \bar{g}+g) /\left(1-|k|^{2}\right)$ is bounded where $k$ and $g$ are functions in $H^{\infty}$ and $|k|<1$ a.e., $T_{\phi}$ is hyponormal.

Proof. A calculation implies that $\phi-k \bar{\phi}=g$ and hence by Lemma $1 T_{\phi}$ is hyponormal.

Theorem 3. Let $\phi$ be a nonzero function in $L^{\infty}$. $T_{\phi}$ is hyponormal if and only if

$$
\phi=\chi_{E}\left(k^{1 / 2} u+\frac{1}{2} g\right)+\chi_{E^{c}} \frac{k \bar{g}+g}{1-|k|^{2}},
$$


where $k$ and $g$ are functions in $H^{\infty}$ with $\|k\|_{\infty} \leq 1, E=\left\{e^{i \theta} \in \Gamma:\left|k\left(e^{i \theta}\right)\right|=\right.$ $1\}, k \bar{g}=-g$ a.e. on $E$ and $u$ is a real-valued function in $L^{\infty}$.

Proof. For the 'if' part, by a simple calculation

$$
\phi-k \bar{\phi}=\chi_{E}\left(\frac{1}{2} g-\frac{1}{2} k \bar{g}\right)+\chi_{E^{c}} g=g
$$

because $k \bar{g}=-g$ a.e. on $E$.

We will show the 'only if' part. Lemma 1 shows that $\phi=k \bar{\phi}+g$ where $g \in H^{\infty}$ and $k \in H^{\infty}$ with $\|k\|_{\infty} \leq 1$. Put $E=\left\{e^{i \theta} \in \Gamma:\left|k\left(e^{i \theta}\right)\right|=1\right\}$. If $g=0$ a.e., then $\phi^{2}=k|\phi|^{2}$ and hence $\phi=\chi_{E} k^{1 / 2} u$ where $u$ is a real-valued function in $L^{\infty}$. We will assume that $g$ is nonzero. Since $\phi=k(\bar{k} \phi+\bar{g})+g$, $\left(1-|k|^{2}\right) \phi=k \bar{g}+g$. This implies that on $E^{c}$

$$
\phi=(k \bar{g}+g) /\left(1-|k|^{2}\right)
$$

and on $E, k \bar{g}+g=0$ and hence $i g=k^{1 / 2}|g|\left(2 \chi_{E_{0}}-1\right)$, where $E_{0}$ is a measurable set of $\Gamma$. Since $\phi g^{-1}-k \bar{\phi} g^{-1}=1$,

$$
\phi / g+\bar{\phi} / \bar{g}=1 \text { on } E
$$

because $k \bar{g}=-g$ on $E$. Then

$$
\phi=(i g) \operatorname{Im}\left(\phi g^{-1}\right)+\frac{1}{2} g=k^{1 / 2} u+\frac{1}{2} g
$$

and $u=|g| \operatorname{Im}\left(\phi g^{-1}\right)\left(2 \chi_{E_{0}}-1\right)$ because $i g=k^{1 / 2}|g|\left(2 \chi_{E_{0}}-1\right)$. This completes the proof.

In Theorem $3 \phi$ has the different forms on $E$ and $E^{c}$. We can expect that $d \theta(E)=2 \pi$ or $d \theta\left(E^{c}\right)=2 \pi$. However this is not the case by the following example. If $k=\exp \left(\chi_{E}-1+i v\right)$ then $\|k\|_{\infty} \leq 1,|k|=1$ a.e. on $E$ and $|k|=$ $e^{-1}$ a.e. on $E^{c}$, where $v$ is a harmonic conjugate of $\chi_{E}$ and $0<d \theta(E)<2 \pi$. Put $g=i k^{1 / 2}$. Then

$$
\phi=\chi_{E}\left(k^{1 / 2}+\frac{1}{2} g\right)+\chi_{E^{c}}(k \bar{g}+g)\left(1-|k|^{2}\right)^{-1}
$$

is bounded and $k \bar{g}+g=0$ on $E$. Moreover,

$$
\phi=k^{1 / 2}\left\{\left(1+\frac{1}{2} i\right) \chi_{E}+i\left(1-e^{-1}\right)\left(1-e^{-2}\right)^{-1} \chi_{E^{c}}\right\} .
$$

Therefore $\phi$ is not of bounded type and hence this representation is unique (see $\S 6$ ). In Proposition 2, if $\|k\|_{\infty}<1$ then $\phi$ is bounded for arbitrary $g$ in $H^{\infty}$. In Proposition 1 , if $q=z$ then $g$ is a polynomial of degree at most one. For arbitrary $k$ in $H^{\infty}$, we want to know such functions $g$. For $k \in H^{\infty}$ with $\|k\|_{\infty} \leq 1$, put

$$
\mathscr{H}(k)=\left\{g \in H^{\infty}: \phi=k \bar{\phi}+g \text { for some } \phi \in L^{\infty}\right\} .
$$

By [5, Theorem 1] we can describe $\mathscr{H}(k)$ as

$$
\mathscr{H}(k)=\left\{g \in H^{\infty}: g=f-k \bar{f}+\overline{T_{\bar{k}} f}-k\left(T_{\bar{k}} f\right) \text { for some } f \in H^{2}\right\} .
$$

The following corollary gives another description of $\mathscr{H}(k)$.

Corollary 1. Let $k$ be in $H^{\infty}$ and $\|k\|_{\infty} \leq 1$. Then $\mathscr{H}(k)$ is a nonzero real subspace of $H^{\infty}$ and

$$
\mathscr{H}(k)=\left\{\alpha g:|g+k \bar{g}|+|k| \leq 1 \text { a.e., } g \in H^{\infty} \text { and } \alpha \in \mathbb{R}\right\} .
$$


If $\|k\|_{\infty}<1$ then $\mathscr{H}(k)=H^{\infty}$ and if $k$ is inner then

$$
\begin{aligned}
\mathscr{H}(k) & =\left\{g \in H^{\infty}: k \bar{g}=-g\right\} \\
& =\left\{g-k \bar{g}: g \in H^{2} \ominus k z H^{2} \text { and } g \in H^{\infty}\right\} .
\end{aligned}
$$

Proof. It is clear that $\mathscr{H}(k)$ is a real subspace and $1-k \in \mathscr{H}(k)$. Since $\left(1-|k|^{2}\right) \phi=k \bar{g}+g$ by the proof of Theorem $3, \mathscr{H}(k)$ has the form above. Hence if $\|k\|_{\infty}<1$ then $\mathscr{H}(k)=H^{\infty}$ trivially. If $k$ is inner then $|g+k \bar{g}| \leq$ $1-|k|=0$ and hence $g=-k \bar{g}$. Thus $\mathscr{H}(k)=\left\{g \in H^{\infty}: k \bar{g}=-g\right\}$. If $g_{0}=g-k \bar{g}$ where $g \in H^{2} \ominus k z H^{2}$ and $g \in H^{\infty}$, then $k \bar{g}_{0}=-g_{0}$ and $g_{0} \in \mathscr{H}(k)$. If $g_{0} \in \mathscr{H}(k)$ then $k \bar{g}_{0}=-g_{0}$ and hence $g_{0} \in H^{2} \ominus k z H^{2}$. Put $g=g_{0} / 2$; then $g_{0}=g-k \bar{g}$ and $g \in H^{2} \ominus k z H^{2}$. Therefore $\mathscr{H}(k)=$ $\left\{g-k \bar{g}: g \in H^{2} \ominus k z H^{2}\right.$ and $\left.g \in H^{\infty}\right\}$.

In the following corollary the equivalence between (1) and (2) is clear by Theorem 3. Statement (3) shows that such a hyponormal Toeplitz operator is the product of a normal Toeplitz (possibly unbounded) and an analytic Toeplitz operator (hence subnormal).

Corollary 2. Let $\phi$ be a function in $L^{\infty}$. Then the following are equivalent.

(1) $\phi=q \bar{\phi}+g$ where $q$ is inner and $g \in H^{\infty}$.

(2) $\phi=q^{1 / 2} u+g$ where $u$ is a real-valued function in $L^{\infty}, q$ is inner, and $g \in H^{\infty}$ with $q \bar{g}=-g$.

(3) $\phi=(v+c) f$ where $v$ is a real-valued measurable function, $c$ is a complex number, and $f \in H^{\infty}$ with $q \bar{f}=f$.

Proof. We will show only the equivalence between (2) and (3).

$(2) \Rightarrow(3)$. For some measurable subset $E$ of $\Gamma$

$$
q^{1 / 2}=i g\left(2 \chi_{E}-1\right) /|g|
$$

because $q \bar{g}=-g$. Put $v=u\left(2 \chi_{E}-1\right) /|g|, f=i g$, and $c=-i$; then $\phi=(v+c) f$.

(3) $\Rightarrow(2)$. Let $c=\alpha+i \beta$ where $\alpha$ and $\beta$ are real. For some measurable subset $E$ of $\Gamma, f /|f|=q^{1 / 2}\left(2 \chi_{E}-1\right)$ because $q \bar{f}=f$. Put $u=$ $|f|(v+\alpha)\left(2 \chi_{E}-1\right)$ and $g=i \beta f ;$ then $\phi=q^{1 / 2} u+g$.

\section{EXtremal PROBlems in HARDY SPACES}

The equation of a hyponormal symbol $\phi: \phi=k \bar{\phi}+g$ is related to the extremal problem in $H^{\infty}$ (and hence $H^{1}$ ) (cf. [8, Chapter IV]) because $\mid \phi-$ $g|\leq| \phi \mid$ a.e. on $\Gamma$. When $\phi=k \bar{\phi}+g$ and $\phi \neq 0$ a.e., the following are equivalent: (1) $g=0$ a.e., (2) $\phi=k \bar{\phi}$, (3) $\phi=k^{1 / 2} u$ where $k$ is inner and $u$ is a real-valued function in $L^{\infty}$, and (4) $\phi=f v$ where $v$ is a real-valued measurable function and $f \in H^{\infty}$ with $k \bar{f}=f$. When does the symbol $\phi$ satisfy one of the equivalent conditions? Such Toeplitz operators have been studied in [4]. We answer this question in the following theorem.

Theorem 4. Let $\phi$ be in $L^{\infty}$ and $\phi \neq 0$ a.e.

(1) When $\log |\phi|$ is not integrable, $T_{\phi}$ is hyponormal if and only if $\phi=q \bar{\phi}$ for some inner $q$. 
(2) Suppose $T_{\phi}$ is hyponormal. If there does not exist a nonzero function $g$ in $H^{\infty}$ with $|\phi-g| \leq|\phi|$ a.e. then $\phi=q \bar{\phi}$ for some inner $q$.

(3) Suppose $T_{\phi}$ is hyponormal and nonnormal. If $\phi=q \bar{\phi}$ for some inner $q$ then there does not exist a nonzero function $g$ in $H^{\infty}$ with $|\phi-\bar{g}| \leq|\phi|$ a.e.

Proof. (1) Suppose $T_{\phi}$ is hyponormal and $\log |\phi|$ is not integrable. If $\phi$ does not have the form $\phi=q \bar{\phi}$ for some inner $q$, then by the remark above Theorem 4 there exists a nonzero function $g$ in $H^{\infty}$ such that $|\phi-g| \leq|\phi|$ a.e. Hence $|g|^{2} \leq 2|\phi||g|$ and $\log |\phi|$ is integrable. This contradiction shows that $\phi=q \bar{\phi}$ for some inner $q$. The converse is just Lemma 1 .

(2) By the proof of (1) if $\phi$ does not have the form $\phi=q \bar{\phi}$ for some inner $q$, then there exists a nonzero function $g$ in $H^{\infty}$ such that $|\phi-g| \leq|\phi|$ a.e.

(3) If there exists a nonzero function $g$ in $H^{\infty}$ with $|\phi-\bar{g}| \leq|\phi|$ a.e., then by the proof of (1) $\log |\phi|$ is integrable and hence there exists an outer function $k$ in $H^{\infty}$ with $|\phi|=|k|$. Put $Q=\bar{\phi} / k$ then $\left|Q+g^{\prime}\right| \leq 1$ a.e. where $g^{\prime}=g / k$. By a lemma of Koosis (cf. [8, pp. 161-163]) there exists an outer function $f$ in $H^{1}$ such that $Q=f /|f|$. If $\phi=q^{1 / 2} u$ where $q$ is inner and $u$ is a real-valued function in $L^{\infty}$ then

$$
q^{1 / 2} u /|u|=\phi /|\phi|=|f k| / f k,
$$

and hence $q(f k)^{2}=|f k|^{2}$. Therefore $q(f k)^{2}$ is a nonnegative function in $H^{1 / 2}$ and hence by [13] $q(f k)^{2}$ is constant. Since $f$ and $k$ are outer, $q$ is constant and $T_{\phi}$ is normal. This contradiction implies (3).

Corollary 3. Suppose $T_{\phi}$ is hyponormal and $\phi \neq 0$ a.e.

(1) If there does not exist an outer function $h$ in $H^{\infty}$ such that $T_{\phi}^{*} T_{\phi} \geq$ $T_{h}^{*} T_{h}$ then $\phi=q \bar{\phi}$ for some inner $q$.

(2) Suppose $T_{\phi}$ is not normal. If there exists an outer function $h$ in $H^{\infty}$ such that $T_{\phi} T_{\phi}^{*} \geq T_{h}^{*} T_{h}$ then $\phi$ does not have the form $\phi=q \bar{\phi}$ for some nonconstant inner $q$.

Proof. By [12, Theorem 2], there exists an outer function $h$ in $H^{\infty}$ such that $T_{\phi}^{*} T_{\phi} \geq T_{h}^{*} T_{h}$ (or $T_{\phi} T_{\phi}^{*} \geq T_{h}^{*} T_{h}$ ) if and only if there exists a nonzero function $g$ in $H^{\infty}$ such that $|\phi-g| \leq|\phi|$ a.e. (or $|\phi-\bar{g}| \leq|\phi|$ a.e.). Now Theorem 4 implies the corollary.

The converses of (2) of Theorem 4 and (1) of Corollary 3 are not true. For example, if $\phi$ is a positive invertible function in $L^{\infty}$ then $\phi=\bar{\phi}$ and $T_{\phi}^{*} T_{\phi}=$ $T_{\phi} T_{\phi}^{*} \geq T_{c}^{*} T_{c}$ for some positive constant $c$ and there exists a nonzero function $g$ in $H^{\infty}$ with $|\phi-g| \leq|\phi|$ a.e. Statement (3) of Theorem 4 (or (2) of Corollary 3 ) is the weak converse of (2) (or (1), respectively) for nonnormal and hyponormal Toeplitz operators. Now we concentrate on unimodular symbols of Toeplitz operators. In the following theorem (3) is known in the different proof [1, Proposition 2].

Theorem 5. Let $\phi$ be a unimodular function in $L^{\infty}$.

(1) If $T_{\phi}$ is hyponormal then $\phi=f /|f|$ for some nonzero function $f$ in $H^{1}$ or $\phi^{2}=q$ for some inner function $q$. 
(2) If $\phi=|f| / f$ for some nonzero function $f$ in $H^{1}$ whose inverse does not belong to $H^{1}$, then $T_{\phi}$ is not hyponormal.

(3) If $T_{\phi}$ is hyponormal and $\phi=\bar{q} b$ where $q$ and $b$ are inner functions, then $\phi=b_{1}$ where $b_{1}$ is an inner divisor of $b$.

(4) Suppose $\phi=f /|f|$ for some nonzero function $f$ in $H^{1}$ and $\bar{f}$ is of bounded type. If $T_{\phi}$ is hyponormal then $\phi^{2}=q$ for some inner $q$.

Proof. (1) If $T_{\phi}$ is hyponormal then by Lemma $1|\phi-g| \leq 1$ a.e. and $\phi(\phi-g) \epsilon$ $H^{\infty}$ for some $g$ in $H^{\infty}$ because $|\phi|=1$ a.e. If $g$ is nonzero then by a theorem of Koosis (cf. [8, pp. 161-163]) $\phi=f /|f|$ for some nonzero $f$ in $H^{1}$. If $g$ is zero then $\phi^{2}=q$ for some inner $q$.

(2) If $T_{\phi}$ is hyponormal then by (1) $|f| / f=h /|h|$ for some nonzero $h$ in $H^{1}$ or $|f|^{2} / f^{2}=q$ for some inner $q$. When $|f| / f=h /|h|, h f$ is a nonnegative function in $H^{1 / 2}$ and when $|f|^{2} / f^{2}=q, q f^{2}$ is a nonnegative function in $H^{1 / 2}$. Hence by [13] $h f$ is constant or $q f^{2}$ is constant. Both contradicts that $f^{-1} \notin H^{1}$.

(3) Put $\bar{q} b=\bar{q}_{1} b_{1}$ where $q_{1}$ and $b_{1}$ are relatively prime inner functions. If $T_{\phi}$ is hyponormal then by Lemma $1 \bar{q}_{1} b_{1}=k q_{1} \bar{b}_{1}+g$ where $k$ and $g$ are in $H^{\infty}$. Therefore

$$
b_{1}^{2}=k q_{1}^{2}+q_{1} b_{1} g=q_{1}\left(k q_{1}+b_{1} g\right)
$$

and hence $q_{1}$ is constant. This implies (3).

(4) If $T_{\phi}$ is hyponormal then by Lemma 1

$$
f=k \bar{f}+g|f|
$$

where $k$ and $g$ are in $H^{\infty}$. If $g$ is zero then $\phi^{2}$ is an inner function. Suppose $g$ is nonzero. If $q$ is an inner function with $q \bar{f} \in H^{1}$ then by the equality above $q g|f|$ belongs to $H^{1}$. Put $f=b h$ and $g=s l$, where $b$ and $s$ are inner and $h$ and $l$ are outer. Then $q s b|f| / f \in H^{\infty}$ and hence $\phi=\bar{Q} q s b$ for some inner $Q$. Now (3) implies (4).

Corollary 4. Put $\phi=f /|f|$ and

$$
f(z)=\prod_{j=1}^{l}\left(z-\alpha_{j}\right) \prod_{j=1}^{m}\left(z-\beta_{j}\right) \prod_{j=1}^{n}\left(z-\gamma_{j}\right)
$$

where $\left|\alpha_{j}\right|<1$ for $1 \leq j \leq l,\left|\beta_{j}\right|=1$ for $1 \leq j \leq m$, and $\left|\gamma_{j}\right|>1$ for $1 \leq j<n$. Then $T_{\phi}$ is hyponormal if and only if $l \geq n$ and $\alpha_{j}^{\prime} \bar{\gamma}_{j}=1$ for $1 \leq j \leq n$ where $\left\{\alpha_{j}^{\prime}\right\}_{j=1}^{n}$ is a subset of $\left\{\alpha_{j}\right\}_{j=1}^{l}$.

Proof. $\phi^{2}=f^{2} /|f|^{2}=f / \bar{f}$ and

$$
\frac{f}{\bar{f}}=\prod_{j=1}^{m}\left(-\beta_{j}\right) \prod_{j=1}^{n} \frac{\gamma_{j}}{\bar{\gamma}_{j}} z^{l+m+n} \prod_{j=1}^{l} \frac{z-\alpha_{j}}{1-\bar{\alpha}_{j} z} \prod_{j=1}^{n} \frac{1-\gamma_{j}^{-1} z}{z-\bar{\gamma}_{j}^{-1}}
$$

By (4) of Theorem 5, $T_{\phi}$ is hyponormal if and only if $\phi^{2}$ is inner if and only if

$$
\prod_{j=1}^{l} \frac{z-\alpha_{j}}{1-\bar{\alpha}_{j} z} \prod_{j=1}^{n} \frac{1-\gamma_{j}^{-1} z}{z-\bar{\gamma}_{j}^{-1}}
$$

belongs to $H^{\infty}$. This implies the corollary. 


\section{ARguments of Symbols}

If $T_{\phi}$ is hyponormal, $|\phi|>0$ a.e., and $\phi_{0}=\phi /|\phi|$ then is $T_{\phi_{0}}$ hyponormal? In this section we answer this question. Statement (3) of Proposition 6 is a generalization of (4) of Theorem 5.

Lemma 2. Suppose $\log |\phi|$ is integrable, $\phi_{0}=\phi /|\phi|$, and $s=h /|h|$ where $h$ is an outer function in $H^{\infty}$ with $|\phi|=|h| . T_{\phi}$ is hyponormal if and only if $\phi_{0}\left(\phi_{0}-s g\right)=k$, where $g$ and $k$ are in $H^{\infty}$, and $\|k\|_{\infty} \leq 1$.

Proof. By Lemma 1, $T_{\phi}$ is hyponormal if and only if $\phi-g_{1}=k \bar{\phi}$ where $g_{1}$ and $k$ are in $H^{\infty}$, and $\|k\|_{\infty} \leq 1$. Since $\phi=\phi_{0} \bar{s} h, \phi-g_{1}=k \bar{\phi}$ if and only if $\phi_{0}\left(\phi_{0}-s g_{1} / h\right)=k$. Since $g_{1} / h$ is in $H^{\infty}$, this implies the lemma.

Proposition 6. Suppose $|\phi|$ is positive almost everywhere and $\phi_{0}=\phi /|\phi|$.

(1) If $T_{\phi}$ is hyponormal and $\log |\phi|$ is not integrable then $T_{\phi_{0}}$ is hyponormal.

(2) Suppose $|\phi|=|h|$ for some outer $h$ in $H^{\infty}$ and $h /|h|$ is inner. If $T_{\phi}$ is hyponormal then $T_{\phi_{0}}$ is hyponormal.

(3) Suppose $\phi$ and $\bar{\phi}$ are of bounded type and $\phi_{0}^{2} \notin H^{\infty}$. Then if $T_{\phi}$ is hyponormal, $T_{\phi_{0}}$ is not hyponormal.

Proof. (1) By (1) of Theorem 4, $\phi=q \bar{\phi}$ for some inner $q$ and hence $\phi_{0}=q \bar{\phi}_{0}$. This implies that $T_{\phi_{0}}$ is hyponormal.

(2) In Lemma $2, s$ is inner by the hypothesis on $h /|h|$ and hence $T_{\phi_{0}}$ is hyponormal.

(3) Under the conditions on $\phi, \bar{\phi}$, and $\phi_{0}$, if both $T_{\phi}$ and $T_{\phi_{0}}$ are hyponormal then we claim a contradiction. By Lemma 1 , there exist four functions $k_{1}, k_{2}, g_{1}$, and $g_{2}$ in $H^{\infty}$ such that $\phi=k_{1} \bar{\phi}+g_{1}$ and $\phi_{0}=k_{2} \bar{\phi}_{0}+g_{2}$. If $g_{2}$ is zero then this contradicts that $\phi_{0}^{2}$ is not inner. If $g_{2}$ is nonzero then

$$
\phi=k_{2} \bar{\phi}+g_{2}|\phi| \text {. }
$$

Since both $\phi$ and $\bar{\phi}$ are of bounded type, $|\phi|$ is also of bounded type and hence $\phi_{0}$ is of bounded type. Therefore $\phi_{0}=\bar{q} b$ where $q$ and $b$ are inner. By (3) of Theorem $5 \phi_{0}$ is inner. This contradicts that $\phi_{0}^{2}$ is not inner, too.

\section{BOUNDED TYPE SYMBOLS}

Abrahamse studied a hyponormal Toeplitz operator $T_{\phi}$ when $\phi$ or $\bar{\phi}$ is of bounded type. He gave several sufficient conditions [1, Proposition 1]. In this section we give necessary conditions, partially solving Problem 1 in [1]. If $\phi$ is of bounded type then $\phi$ has the form $\phi=\overline{q_{1}} q_{2} h$ where $q_{1}$ and $q_{2}$ are relatively prime inner functions, and $h$ is outer. We call $q_{0}$ a minimal inner function for $\phi$ when $q_{0}$ is inner and it satisfies the following: if $q$ is inner with $q \phi \in H^{\infty}$ then $\overline{q_{0}} q \in H^{\infty}$. In fact $q_{1}$ is the minimal inner function for $\phi$.

Proposition 7. Suppose $\phi$ is of bounded type, that is, $\phi$ has the form $\phi=\overline{q_{1}} q_{2} h$ where $q_{1}$ and $q_{2}$ are relatively prime inner functions, and $h$ is outer.

(1) If $T_{\phi}$ is hyponormal then $h / \bar{h}$ has the form $h / \bar{h}=\bar{F} G$ where $F$ and $G$ are inner, and $G \bar{q}_{1}^{2}$ is in $H^{\infty}$. 
(2) If $h / \bar{h}=\bar{F} G$ where $F$ and $G$ are inner, and both $G \bar{q}_{1}^{2}$ and $\bar{F} q_{2}^{2}$ are in $H^{\infty}$, then $T_{\phi}$ is hyponormal.

Proof. (1) By Lemma 1 and the hypothesis on $\phi, \bar{q}_{1} q_{2} h-g=k q_{1} \bar{q}_{2} \bar{h}$ where $g$ and $k$ are in $H^{\infty}$ and hence'

$$
q_{2}^{2}-q_{1} q_{2} g / h=k q_{1}^{2} \bar{h} / h=k q_{1}^{2} \bar{G} F
$$

where $G$ and $F$ are inner. Therefore $G\left(q_{2}^{2}-q_{1} q_{2} g / h\right)=k q_{1}^{2} F$ and hence $G \bar{q}_{1}^{2} \in H^{\infty}$ becasue $q_{1}$ and $q_{2}$ are relatively prime.

(2) If $h / \bar{h}=\bar{F} G$ then by the hypothesis on $F$ and $G$,

$$
\frac{\phi}{\bar{\phi}}=\frac{\bar{q}_{1} q_{2} h}{q_{1} \bar{q}_{2} \bar{h}}=\left(\bar{q}_{1}^{2} G\right)\left(q_{2}^{2} \bar{F}\right) \in H^{\infty} .
$$

Lemma 1 implies that $T_{\phi}$ is hyponormal.

Corollary 5. Let $m$ and $n$ be nonnegative integers. Suppose $\phi=\sum_{j=-n}^{m} a_{j} z^{j}$, $a_{-n} \neq 0$, and $a_{m} \neq 0$; then the following hold.

(1) If $T_{\phi}$ is hyponormal then $m \geq n$.

(2) Suppose $m \geq n$ and if $z^{n} \phi$ is zero on a point $z_{0}$ outside the closed unit disc, then it is zero on the inverse of $\bar{z}_{0}$ with higher multiplicity. Then $T_{\phi}$ is hyponormal.

(3) Suppose $m=n$ and $T_{\phi}$ is not normal. If $z^{n} \phi$ does not have any zeros in the open unit disc then $T_{\phi}$ is not hyponormal.

(4) Suppose $m=n$ and if $z^{n} \phi$ has zeros only on the unit circle, then $T_{\phi}$ is normal.

Proof. Put $k=z^{n} \phi=q h$ where $q$ is inner and $h$ is outer, and write

$$
k=\prod_{j=1}^{l}\left(z-\alpha_{j}\right) \prod_{j=1}^{t}\left(z-\beta_{j}\right) \prod_{j=1}^{s}\left(z-\gamma_{j}\right)
$$

where $\left|\alpha_{j}\right|<1$ for $1 \leq j \leq l,\left|\beta_{j}\right|=1$ for $1 \leq j \leq t$, and $\left|\gamma_{j}\right|>1$ for $1 \leq j \leq s$. Then

$$
q=\prod_{j=1}^{l} \frac{z-\alpha_{j}}{1-\bar{\alpha}_{j} z}
$$

and

$$
h=\prod_{j=1}^{l}\left(1-\bar{\alpha}_{j} z\right) \prod_{j=1}^{t}\left(z-\beta_{j}\right) \prod_{j=1}^{s}\left(z-\gamma_{j}\right),
$$

and the degree of $k=m+n=l+t+s$. Hence

$$
\frac{h}{\bar{h}}=\prod_{j=1}^{t}\left(-\beta_{j}\right) \prod_{j=1}^{s} \frac{\gamma_{j}}{\bar{\gamma}_{j}} z^{m+n} \prod_{j=1}^{l} \frac{1-\bar{\alpha}_{j} z}{z-\alpha_{j}} \prod_{j=1}^{s} \frac{1-\gamma_{j}^{-1} z}{z-\bar{\gamma}_{j}^{-1}} .
$$

(1) If $T_{\phi}$ is hyponormal then by (1) of Proposition $7 z^{m+n} / z^{2 n}$ is analytic and hence $m \geq n$.

(2) By the hypothesis $\alpha_{j}^{\prime} \bar{\gamma}_{j}=1$ for $1 \leq j \leq s \leq l$ where $\left\{\alpha_{j}^{\prime}\right\}_{j=1}^{l}=\left\{\alpha_{j}\right\}_{j=1}^{l}$, and hence

$$
\left(\prod_{j=1}^{s} \frac{1-\gamma_{j}^{-1} z}{z-\bar{\gamma}_{j}^{-1}}\right) q \in H^{\infty} .
$$


Then (2) of Proposition 7 implies (2).

(3) If $m=n$ and $k=\prod_{j=1}^{t}\left(z-\beta_{j}\right) \prod_{j=1}^{s}\left(z-\gamma_{j}\right)$ then

$$
\bar{\phi}=z^{n} \prod_{j=1}^{t}\left(\bar{z}-\bar{\beta}_{j}\right) \prod_{j=1}^{s}\left(\bar{z}-\bar{\gamma}_{j}\right)=\bar{z}^{n} k^{\prime}
$$

where

$$
k^{\prime}=\prod_{j=1}^{t}\left(1-\bar{\beta}_{j} z\right) \prod_{j=1}^{s}\left(1-\bar{\gamma}_{j} z\right) .
$$

By (2) $T_{\phi}^{*}$ is hyponormal and hence if $T_{\phi}$ is not normal then it is not hyponormal.

(4) is clear by (2) and (3)

When $\phi=a_{-1} \bar{z}+a_{0}+a_{1} z$ and $a_{-1} \neq 0$, it is easy to see that $T_{\phi}$ is hyponormal if and only if $\left|a_{1}\right| \geq\left|a_{-1}\right|$. When $\phi=\bar{z}(z-\alpha)(z-\gamma)$ and $\alpha \gamma \neq 0, T_{\phi}$ is hyponormal if and only if $|\alpha \gamma| \leq 1$. Therefore the question about polynomials is still open.

Corollary 6. Suppose $\phi$ is a rational function in $L^{\infty}: \phi=k_{2} / k_{1}$ where $k_{1}$ and $k_{2}$ are relatively prime analytic polynomials with the same degree. Write

$$
k_{1}=\prod_{j=1}^{l}\left(z-a_{j}\right) \prod_{j=1}^{n}\left(z-c_{j}\right)
$$

where $\left|a_{j}\right|<1$ for $1 \leq j \leq l$ and $\left|c_{j}\right|>1$ for $1 \leq j \leq n$, and

$$
k_{2}=\prod_{j=1}^{d}\left(z-\alpha_{j}\right) \prod_{j=1}^{e}\left(z-\beta_{j}\right) \prod_{j=1}^{f}\left(z-\gamma_{j}\right)
$$

where $\left|\alpha_{j}\right|<1$ for $1 \leq j \leq d,\left|\beta_{j}\right|=1$ for $1 \leq j \leq e$, and $\left|\gamma_{j}\right|>1$ for $1 \leq$ $j \leq f$. If $T_{\phi}$ is hyponormal then $\left\{\dot{a}_{j}^{-1}\right\}_{j=1}^{l} \subseteq\left\{\bar{c}_{j}\right\}_{j=1}^{n}$. If $\left\{a_{j}^{-1}\right\}_{j=1}^{l} \subseteq\left\{\bar{c}_{j}\right\}_{j=1}^{n}$ and $\left\{\alpha_{j}^{-1}\right\}_{j=1}^{d} \supset\left\{\bar{\gamma}_{j}\right\}_{j=1}^{f}$ then $T_{\phi}$ is hyponormal.

Proof. Suppose $k_{j}=q_{j} h_{j}(j=1,2)$ are inner outer factorizations. Then

$$
q_{1}=\prod_{j=1}^{l} \frac{z-a_{j}}{1-\bar{a}_{j} z}, \quad h_{1}=\prod_{j=1}^{l}\left(1-\bar{a}_{j} z\right) \prod_{j=1}^{n}\left(z-c_{j}\right)
$$

and

$$
q_{2}=\prod_{j=1}^{d} \frac{z-\alpha_{j}}{1-\bar{\alpha}_{j} z}, \quad h_{2}=\prod_{j=1}^{d}\left(1-\bar{\alpha}_{j} z\right) \prod_{j=1}^{e}\left(z-\beta_{j}\right) \prod_{j=1}^{f}\left(z-\gamma_{j}\right),
$$

$\phi=\bar{q}_{1} q_{2} h$ and $h=h_{2} / h_{1}$. Since

$$
\frac{\bar{h}_{1}}{h_{1}}=A \bar{z}^{l+n} q_{1} \prod_{j=1}^{n} \frac{z-\bar{c}_{j}^{-1}}{1-c_{j}^{-1} z}
$$

and

$$
\frac{h_{2}}{\bar{h}_{2}}=B z^{d+e+f} \bar{q}_{2} \prod_{j=1}^{f} \frac{1-\gamma_{j}^{-1} z}{z-\bar{\gamma}_{j}^{-1}}
$$


where $A$ and $B$ are constants, $l+n=d+e+f, h / \bar{h}=\bar{F} G$, and

$$
\bar{F}=C \bar{q}_{2} \prod_{j=1}^{f} \frac{1-\gamma_{j}^{-1} z}{z-\bar{\gamma}_{j}^{-1}} \quad \text { and } \quad G=q_{1} \prod_{j=1}^{n} \frac{z-\bar{c}_{j}^{-1}}{1-c_{j}^{-1} z}
$$

where $C$ is a constant with absolute value 1 . Now Proposition 7 implies the corollary.

\section{Self-Commutator of $T_{\phi}$}

For $\phi$ in $L^{\infty}$, put

$$
\mathscr{E}(\phi)=\left\{k \in H^{\infty}: \phi=k \bar{\phi}+g, g \in H^{\infty}, \text { and }\|k\|_{\infty} \leq 1\right\} .
$$

If $\mathscr{E}(\phi)$ contains an inner function $q$ then by Corollary $2 \phi$ has a simple form. In this section we study when $\mathscr{E}(\phi)$ contains an inner function.

Proposition 8. Suppose $\mathscr{E}(\phi)$ contains at least two elements. Then the following are valid.

(1) $\phi$ is of bounded type.

(2) For any fixed function $k$ in $\mathscr{E}(\phi)$

$$
\mathscr{E}(\phi)=\left\{k+q_{0} f:\left\|k+q_{0} f\right\|_{\infty} \leq 1 \text { and } f \in H^{\infty}\right\}
$$

where $q_{0}$ is the minimal inner function for $\bar{\phi}$.

(3) There exists an inner function $b$ in $\mathscr{E}(\phi)$.

Proof. If $k_{1}$ and $k_{2}$ are in $\mathscr{E}(\phi)$ and $k_{1} \neq k_{2}$ then $\phi=k_{1} \bar{\phi}+g_{1}=k_{2} \bar{\phi}+g_{2}$ for some $g_{1}$ and $g_{2}$ in $H^{\infty}$. Hence $\left(k_{1}-k_{2}\right) \bar{\phi}=g_{2}-g_{1}$ and this implies (1). Since $\left(k_{1}-k_{2}\right) \bar{\phi} \in H^{\infty}, q \bar{\phi}$ belongs to $H^{\infty}$ where $q$ is the inner part of $k_{1}-k_{2}$. If $q_{0}$ is the minimal inner function for $\bar{\phi}$ then $\bar{q}_{0} q \in H^{\infty}$ and hence $\bar{q}_{0}\left(k_{1}-k_{2}\right) \in H^{\infty}$. This implies $(2)$. If $\mathscr{E}(\phi)$ contains at least two functions then by (2) and a well-known theorem of Adamyan, Arov, and Krein (cf. [8, Theorem 5.3]) there exists an inner function in $\mathscr{E}(\phi)$.

We use Hankel operators. For $\phi \in L^{\infty}$, the Hankel operator $H_{\phi}: H^{2} \rightarrow$ $L^{2} \ominus H^{2}$ is defined by $H_{\phi} f=(I-P)(\phi f)$ for $f \in H^{2}$, where $P$ is the orthogonal projection of $L^{2}$ onto $H^{2}$ (cf. [14]).

Proposition 9. Suppose $\phi$ is not bounded type and $T_{\phi}$ is hyponormal. If

$$
\operatorname{Ker}\left[T_{\phi}^{*}, T_{\phi}\right] \neq\{0\}
$$

then there exists an inner function in $\mathscr{E}(\phi)$.

Proof. Suppose $x \in \operatorname{Ker}\left[T_{\phi}^{*}, T_{\phi}\right]$ and $k \in \mathscr{E}(\phi)$. Then $\left\|H_{\bar{\phi}} x\right\|_{2}=\left\|H_{\phi} x\right\|_{2}$ because $T_{\phi}^{*} T_{\phi}-T_{\phi} T_{\phi}^{*}=H_{\bar{\phi}}^{*} H_{\bar{\phi}}-H_{\phi}^{*} H_{\phi}$ and

$$
\left\|H_{\phi} x\right\|_{2}=\left\|H_{k \bar{\phi}} x\right\|_{2} \leq\left\|k H_{\bar{\phi}} x\right\|_{2} \leq\left\|H_{\bar{\phi}} x\right\|_{2} .
$$

Since $\phi$ is not of bounded type, $\left\|H_{\phi} x\right\| \neq 0$ and hence $|k|=1$ a.e.

The following lemma is known and is easy to prove. 
Lemma 3. For $k \in \mathscr{E}(\phi)$,

$$
\left[T_{\phi}^{*}, T_{\phi}\right]=H_{\bar{\phi}}^{*}\left(1-J T_{\tilde{k}} T_{\tilde{k}}^{*} J\right) H_{\bar{\phi}}
$$

where $\tilde{k}(z)=\overline{k(\bar{z})}$ and $(J x)(z)=x(\bar{z}) \bar{z}$ for $x$ in $L^{2}$.

Theorem 10. $T_{\phi}$ is hyponormal and $\left[T_{\phi}^{*}, T_{\phi}\right]$ is a finite rank operator if and only if there exists a finite Blaschke product $b$ in $\mathscr{E}(\phi)$. Then we can choose $b$ such that the degree of $b=\operatorname{rank}\left[T_{\phi}^{*}, T_{\phi}\right]$.

Proof. If $b \in \mathscr{E}(\phi)$ is a finite Blaschke product of degree $n$ then

$$
\operatorname{rank}\left(1-J T_{\tilde{b}} T_{\tilde{b}}^{*} J \mid \bar{z} \bar{H}^{2}\right) \leq n
$$

and hence by Lemma $3 \operatorname{rank}\left[T_{\phi}^{*}, T_{\phi}\right] \leq n$. We will show the existence of a finite Blaschke product $b$ in $\mathscr{E}(\phi)$ of degree $n$, assuming that $k \in \mathscr{E}(\phi)$ and $\operatorname{rank}\left[T_{\phi}^{*}, T_{\phi}\right]=n<\infty$. If $\bar{\phi}$ is not of bounded type then $\operatorname{Ran} H_{\bar{\phi}}$ is dense in $\bar{z} \bar{H}^{2}$ and hence by Lemma $3 \operatorname{rank}\left[T_{\phi}^{*}, T_{\phi}\right]=\operatorname{rank}\left(1-J T_{\tilde{k}} T_{\tilde{k}}^{*} J \mid \bar{z} \bar{H}^{2}\right)$. Hence $\tilde{k}$ is a finite Blaschke product of degree $n$ and so is $k$. Now we assume that $\bar{\phi}$ is of bounded type. Then by Beurling's theorem $\operatorname{Ker} H_{\bar{\phi}}=q H^{2}$ for some inner $q$. By Lemma $3 \operatorname{Ker}\left[T_{\phi}^{*}, T_{\phi}\right] \supseteq \operatorname{Ker} H_{\bar{\phi}}$.

Case (i). $\operatorname{Ker}\left[T_{\phi}^{*}, T_{\phi}\right]=\operatorname{Ker} H_{\bar{\phi}}$. Then the closure of $\operatorname{Ran}\left[T_{\phi}^{*}, T_{\phi}\right]=H^{2} \ominus$ $q H^{2}$ and hence

$$
\operatorname{dim}\left(H^{2} \ominus q H^{2}\right)=\operatorname{rank}\left[T_{\phi}^{*}, T_{\phi}\right]=n<\infty .
$$

Therefore $q$ is a finite Blaschke product of degree $n$. By Pick's theorem (cf. [8, Theorem 2.2]), there exists a finite Blaschke product $b$ such that $b \in k+q H^{\infty}$ and the degree of $b$ is at most $n$. Ker $H_{\bar{\phi}}=q H^{2}$ implies that $q$ is the minimal inner function for $\bar{\phi}$, and hence by (2) of Proposition $8 b$ belongs to $\mathscr{E}(\phi)$.

Case (ii). $\operatorname{Ker}\left[T_{\phi}^{*}, T_{\phi}\right] \supsetneq \operatorname{Ker} H_{\bar{\phi}}$. Then there exists a function $x$ in $\operatorname{Ker}\left[T_{\phi}^{*}, T_{\phi}\right]$ such that $\left\|H_{\bar{\phi}} x\right\| \neq 0$. The proof of Proposition 9 implies that $k$ is inner. Since $\operatorname{Ker} H_{\bar{\phi}}^{*}=\overline{q z} \bar{H}^{2}=$ the orthogonal complement of $\operatorname{Ran} H_{\bar{\phi}}$ in $\bar{z} \bar{H}^{2}$, by Lemma 3

$$
\begin{aligned}
\operatorname{rank}\left[T_{\phi}^{*}, T_{\phi}\right] & =\operatorname{dim}\left(1-J T_{\tilde{k}} T_{\tilde{k}}^{*} J\right) H_{\bar{\phi}} H^{2} \\
& =\operatorname{dim}\left(1-T_{\tilde{k}} T_{\tilde{k}}^{*}\right)\left(H^{2} \ominus \tilde{q} H^{2}\right) \\
& =\operatorname{dim}\left\{H^{2} \ominus \tilde{q} H^{2} /\left(H^{2} \ominus \tilde{q} H^{2}\right) \cap \tilde{k} H^{2}\right\} .
\end{aligned}
$$

By a theorem of the first author [11], $k$ is a finite Blaschke product of degree $n$.

Corollary 7. If $\phi$ is a trigonometric polynomial and $T_{\phi}$ is hyponormal, then there exists a finite Blaschke product in $\mathscr{E}(\phi)$.

\section{KERNEL OF THE SELF-COMMUTATOR OF $T_{\phi}$}

In this section we are interested in describing $\operatorname{Ker}\left[T_{\phi}^{*}, T_{\phi}\right]$ when $T_{\phi}$ is hyponormal. $\mathscr{E}(\phi) \ni 0$ if and only if $\phi \in H^{\infty}$. Then $\operatorname{Ker}\left[T_{\phi}^{*}, T_{\phi}\right]=\{0\}$ or 
$\operatorname{Ker}\left[T_{\phi}^{*}, T_{\phi}\right]=q H^{2}$ for some inner function $q$. Proposition 11 is a generalization of this. There is a constant $\beta$ in $\mathscr{E}(\phi)$ with $|\beta|=1$ if and only if $\phi=\beta^{1 / 2} u+\alpha$ where $\beta$ and $\alpha$ are constants and $|\beta|=1$. Then $\operatorname{Ker}\left[T_{\phi}^{*}, T_{\phi}\right]=H^{2}$. Proposition 12 is a generalization of this.

Proposition 11. If there exists a function in $\mathscr{E}(\phi)$ which is not inner, then $\operatorname{Ker}\left[T_{\phi}^{*}, T_{\phi}\right]=q H^{2}$ for some inner $q$ or $\operatorname{Ker}\left[T_{\phi}^{*}, T_{\phi}\right]=\{0\}$. Hence if $\mathscr{E}(\phi)$ contains at least two functions then $\operatorname{Ker}\left[T_{\phi}^{*}, T_{\phi}\right]=q H^{2}$.

Proof. Let $k$ be not an inner function in $\mathscr{E}(\phi)$. Then $\operatorname{Ker}\left(1-T_{\tilde{k}} T_{\tilde{k}}^{*}\right)=\{0\}$ because $\tilde{k}$ is not inner. Hence by Lemma $3 \operatorname{Ker}\left[T_{\phi}^{*}, T_{\phi}\right]=\operatorname{Ker} H_{\bar{\phi}}$ and this implies the proposition.

Proposition 12. If there exists an inner function $q$ in $\mathscr{E}(\phi)$ then

$$
\operatorname{Ker}\left[T_{\phi}^{*}, T_{\phi}\right]=\left\{x \in H^{2}: T_{\bar{\phi}}(q x) \in q H^{2}\right\}=\operatorname{Ker}\left(T_{\bar{\phi}} T_{q}-T_{q} T_{\bar{\phi}}\right) .
$$

Proof. It is sufficient to prove that $\operatorname{Ran}\left[T_{\phi}^{*}, T_{\phi}\right]$ is dense in $T_{\phi \bar{q}}\left(H^{2} \ominus q H^{2}\right)$. Since

$$
\begin{aligned}
H_{\bar{\phi}}^{*} J\left(1-T_{\tilde{q}} T_{\tilde{q}}^{*}\right) H^{2} & =H_{\bar{\phi}}^{*} J\left(H^{2} \ominus \tilde{q} H^{2}\right)=P \phi\left(\bar{z} \bar{H}^{2} \ominus \overline{q z} \bar{H}^{2}\right) \\
& =P \phi \bar{q}\left(H^{2} \ominus q H^{2}\right)=T_{\phi \bar{q}}\left(H^{2} \ominus q H^{2}\right),
\end{aligned}
$$

by Lemma $3 \operatorname{Ran}\left[T_{\phi}^{*}, T_{\phi}\right]$ is dense in $T_{\phi \bar{q}}\left(H^{2} \ominus q H^{2}\right)$. The second equality is trivial.

Lemma 4. $H_{\phi}^{*} H_{\phi}=H_{\psi}^{*} H_{\psi}$ if and only if $\phi-c \psi \in H^{\infty}$ for some constant $c$ with $|c|=1$.

Proof. If $H_{\psi}^{*} H_{\psi}-H_{\phi}^{*} H_{\phi} \geq 0$ then by the proof of [5, Theorem 1] there exist two functions $k$ and $g$ in $H^{\infty}$ with $\|k\|_{\infty} \leq 1$ such that $\phi=k \psi+g$. Lemma 1 is the special case: $\psi=\bar{\phi}$. Hence $H_{\phi}^{*} H_{\phi}=H_{\psi}^{*} H_{\psi}$ then there exist two other functions $h$ and $f$ in $H^{\infty}$ with $\|h\|_{\infty} \leq 1$ such that $\psi=h \phi+f$. Then $(1-k h) \phi=k f+g$. If $1-k h$ is nonzero then $1-k h$ is outer and hence $\phi$ belongs to $H^{\infty}$. Similarly $\psi \in H^{\infty}$. If $1=k h$ then both $k$ and $h$ are constants with $|k|=|h|=1$. Hence $\phi-c \psi \in H^{\infty}$ for some constant $c$ with $|c|=1$. The converse is clear.

Theorem 13. Let $T_{\phi}$ be hyponormal. Then $\operatorname{Ker}\left[T_{\phi}^{*}, T_{\phi}\right] \supseteq q H^{2}$ for some inner function $q$ if and only if $\bar{\phi}$ is of bounded type or $T_{\phi}$ is normal. Moreover if $\operatorname{Ker}\left[T_{\phi}^{*}, T_{\phi}\right] \supseteq q H^{2}$, and $T_{\phi}$ is not normal then $\operatorname{Ker} H_{\bar{\phi}} \supseteq q H^{2}$.

Proof. By Lemma $3 \operatorname{Ker}\left[T_{\phi}^{*}, T_{\phi}\right] \supseteq \operatorname{Ker} H_{\bar{\phi}}$ and hence the 'if' part is clear. We will show the 'only if' part. If $\operatorname{Ker}\left[T_{\phi}^{*}, T_{\phi}\right] \supseteq q H^{2}$ then

$$
0=T_{q}^{*}\left[T_{\phi}^{*}, T_{\phi}\right] T_{q}=T_{\phi q}^{*} T_{\phi q}-T_{\bar{\phi} q}^{*} T_{\bar{\phi} q}=H_{\bar{\phi} q}^{*} H_{\bar{\phi} q}-H_{\phi q}^{*} H_{\phi q} .
$$

By Lemma $4 \bar{\phi} q-c \phi q \in H^{\infty}$ for some constant $c$ with $|c|=1$. Since $T_{\phi}$ is hyponormal, $\phi-k \bar{\phi}=g \in H^{\infty}$ for some $k \in H^{\infty}$ with $\|k\|_{\infty} \leq 1$. Hence $\bar{\phi}(1-c k) q \in H^{\infty}$. If $\bar{\phi}$ is not of bounded type then $1-c k=0$ and hence $k=\bar{c}$. By the remark above Lemma $4, T_{\phi}$ is normal. If $T_{\phi}$ is not normal then $1-c k$ is nonzero and hence $1-c k$ is outer. Therefore $\bar{\phi} q \in H^{2}$ and hence Ker $H_{\bar{\phi}} \supseteq q H^{2}$. 


\section{SUbNORMAL TOEPLITZ OPERATOR}

In this section we will give a simple proof of the Abrahamse's theorem [1] which gives a sufficient condition for the positive answer of Halmos's question [9]. Then we will consider subnormal Toeplitz operators with selfcommutator of finite rank.

Theorem 14 [Abrahamse]. If $T_{\phi}$ is subnormal and if $\phi$ or $\bar{\phi}$ is of bounded type then $T_{\phi}$ is normal or analytic.

Proof. If $T_{\phi}$ is subnormal then by Lemma $1 \phi=k \bar{\phi}+g, k \in H^{\infty}$, and $g \in H^{\infty}$. If $\phi \notin H^{\infty}$ and $\phi$ is of bounded type then $k$ is nonzero and hence $\bar{\phi}$ is also of bounded type (see [1, Lemma 6]). Therefore, we may assume that $\bar{\phi}$ is of bounded type. If $T_{\phi}$ is nonnormal then $q H^{2}=\operatorname{Ker} H_{\bar{\phi}} \subseteq \operatorname{Ker}\left[T_{\phi}^{*}, T_{\phi}\right]$ and $q$ is a nonconstant inner function. $T_{\phi} \operatorname{Ker}\left[T_{\phi}^{*}, T_{\phi}\right] \subseteq \operatorname{Ker}\left[T_{\phi}^{*}, T_{\phi}\right]$ because $T_{\phi}$ is subnormal [1], and hence $T_{\phi}\left(q H^{2}\right) \subseteq \operatorname{Ker}\left[T_{\phi}^{*}, T_{\phi}\right]$. While $\phi q \in H^{\infty}$ because $\operatorname{Ker} H_{\phi} \supseteq \operatorname{Ker} H_{\bar{\phi}}$, and hence the closure of $\phi q H^{2}$ is an invariant subspace in $\operatorname{Ker}\left[T_{\phi}^{*}, T_{\phi}\right]$. By Beurling's theorem and Theorem 13, $\phi q H^{2} \subseteq \operatorname{Ker} H_{\bar{\phi}}=q H^{2}$ and hence $\phi \in H^{\infty}$.

Recall that a Toeplitz operator $T_{\phi}$ is hyponormal and $\operatorname{Ran}\left[T_{\phi}^{*}, T_{\phi}\right]<\infty$ if and only if $\phi=q \bar{\phi}+g$ where $q$ is a finite Blaschke product and $g \in H^{\infty}$. The proof of Proposition 12 shows the following lemma.

Lemma 5. If $\phi=q \bar{\phi}+g$ where $q$ is inner and $g \in H^{\infty}$ then the closure of $\operatorname{Ran}\left[T_{\phi}^{*}, T_{\phi}\right]$ equals the closure of $T_{\phi \bar{q}}\left(H^{2} \ominus q H^{2}\right)$.

Lemma 6. Suppose $\phi=q \bar{\phi}+g$ where $q$ is inner and $g \in H^{\infty}$, and $T_{\phi}$ is subnormal.

(1) Put $M=$ the closure of $\operatorname{Ran}\left[T_{\phi}^{*}, T_{\phi}\right]+\left(H^{2} \ominus q H^{2}\right)$ then $M$ is a $T_{\bar{\phi}}$-invariant subspace.

(2) If $\bar{\phi}$ is not of bounded type then $\operatorname{Ran}\left[T_{\phi}^{*}, T_{\phi}\right] \cap\left(H^{2} \ominus q H^{2}\right)=\{0\}$.

Proof. (1) Since $\phi=q \bar{\phi}+g, T_{\bar{\phi}}\left(H^{2} \ominus q H^{2}\right) \subseteq T_{\bar{q} \phi}\left(H^{2} \ominus q H^{2}\right)+T_{\bar{g}}\left(H^{2} \ominus q H^{2}\right)$. Hence by Lemma 5

$$
T_{\bar{\phi}}\left(H^{2} \ominus q H^{2}\right) \subseteq \text { the closure of } \operatorname{Ran}\left[T_{\phi}^{*}, T_{\phi}\right]+\left(H^{2} \ominus q H^{2}\right) .
$$

This implies (1) because the closure of $\operatorname{Ran}\left[T_{\phi}^{*}, T_{\phi}\right]$ is a $T_{\bar{\phi}}$-invariant subspace.

(2) Since $\operatorname{Ran}\left[T_{\phi}^{*}, T_{\phi}\right] \subseteq T_{\phi \bar{q}}\left(H^{2} \ominus q H^{2}\right)$ by the proof of Lemma 5 , it is sufficient to prove that

$$
T_{\phi \bar{q}}\left(H^{2} \ominus q H^{2}\right) \cap\left(H^{2} \ominus q H^{2}\right)=\{0\} .
$$

If $x \in T_{\phi \bar{q}}\left(H^{2} \ominus q H^{2}\right) \cap\left(H^{2} \ominus q H^{2}\right)$ then $x=T_{\phi \bar{q}} y$ for some $y \in H^{2} \ominus q H^{2}$. Since $T_{\bar{q}} x=0$ because $x \in H^{2} \ominus q H^{2}$,

$$
T_{\phi \bar{q}} 2 y=T_{\bar{q}} T_{\phi \bar{q}} y=T_{\bar{q}} x=0,
$$

and hence $\phi \bar{q}^{2} y=\overline{z k}$ for some $k \in H^{2}$. Therefore $\bar{\phi} q(q \bar{y})=z k \in H^{2}$ and $q \bar{y} \in H^{2}$. If $\bar{\phi}$ is not of bounded type then $y=0$ and hence $x=0$. This implies (2). 
Theorem 15. If $T_{\phi}$ is subnormal and $\phi=q \bar{\phi}$ where $q$ is a finite Blaschke product, then $T_{\phi}$ is normal or analytic.

Proof. By Abrahamse's theorem we may assume that $\bar{\phi}$ is not of bounded type. Under this assumption, we show that $q$ is constant and so $T_{\phi}$ is normal. Since $\phi=q \bar{\phi}$ and $q$ is a finite Blaschke product, by Lemma 5

$$
\operatorname{Ran}\left[T_{\phi}^{*}, T_{\phi}\right]=T_{\phi \bar{q}}\left(H^{2} \ominus q H^{2}\right)=T_{\bar{\phi}}\left(H^{2} \ominus q H^{2}\right) .
$$

Since $T_{\phi}$ is subnormal, $T_{\bar{\phi}} \operatorname{Ran}\left[T_{\phi}^{*}, T_{\phi}\right] \subseteq \operatorname{Ran}\left[T_{\phi}^{*}, T_{\phi}\right]$ and so

$$
T_{\bar{\phi}}\left\{\operatorname{Ran}\left[T_{\phi}^{*}, T_{\phi}\right]+\left(H^{2} \ominus q H^{2}\right)\right\}=\operatorname{Ran}\left[T_{\phi}^{*}, T_{\phi}\right] .
$$

Then by (2) of Lemma 6

$$
\operatorname{dim} \operatorname{Ker} T_{\bar{\phi}} \mid\left\{\operatorname{Ran}\left[T_{\phi}^{*}, T_{\phi}\right]+\left(H^{2} \ominus q H^{2}\right)\right\}=\operatorname{dim}\left(H^{2} \ominus q H^{2}\right),
$$

and hence $\operatorname{dim} \operatorname{Ker} T_{\bar{\phi}} \geq \operatorname{dim}\left(H^{2} \ominus q H^{2}\right)$. The relation $\phi=q \bar{\phi}$ also implies

$$
T_{\phi}\left(\operatorname{Ker} T_{\bar{\phi}}\right) \subseteq \operatorname{Ker} T_{\bar{q}}=H^{2} \ominus q H^{2} .
$$

Since $T_{\phi}$ is hyponormal, $\operatorname{Ker} T_{\bar{\phi}} \supseteq \operatorname{Ker} T_{\phi}$ and $\operatorname{Ker} T_{\phi}=\{0\}$ by Coburn's theorem [7, Proposition 7.24]. Therefore we have $T_{\phi}\left(\operatorname{Ker} T_{\bar{\phi}}\right)=H^{2} \ominus q H^{2}$ and so $\operatorname{Ran} T_{\phi} \supseteq H^{2} \ominus q H^{2}$. Then, noting the relation $T_{\phi} T_{q}-T_{q} T_{\phi}=H_{q}^{*} H_{\phi}$ and $\operatorname{Ran} H_{\bar{q}}^{*}=H^{2} \ominus q H^{2}$, we see that the inclusion $\operatorname{Ran} T_{\phi} \supseteq q^{n}\left(H^{2} \ominus q H^{2}\right)$ implies $\operatorname{Ran} T_{\phi} \supseteq q^{n+1}\left(H^{2} \ominus q H^{2}\right)$ for $n=0,1,2, \ldots$ Thus we have $\operatorname{Ran} T_{\phi} \supseteq$ $\bigcup_{n \geq 0} q^{n}\left(H^{2} \ominus q H^{2}\right)$. Suppose $q$ is not constant. Then $\bigvee_{n \geq 0} q^{n}\left(H^{2} \ominus q H^{2}\right)=H^{2}$ and $T_{\phi}$ has dense range. On the other hand, the relation $T_{\phi}\left(\operatorname{Ker} T_{\bar{\phi}}\right)=H^{2} \ominus q H^{2}$ implies $\operatorname{Ker} T_{\bar{\phi}} \neq\{0\}$. This is a contradiction. We conclude that $q$ is constant.

\section{REFERENCES}

1. M. B. Abrahamse, Subnormal Toeplitz operators and functions of bounded type, Duke Math. J. 43 (1976), 597-604.

2. I. Amemiya, T. Ito, and T. K. Wong, On quasinormal Toeplitz operators, Proc. Amer. Math. Soc. 50 (1975), 254-258.

3. A. Brown and P. R. Halmos, Algebraic properties of Toeplitz operators, J. Reine Angew. Math. 213 (1963/64), 89-102.

4. K. F. Clancey and B. B. Morrel, The essential spectrum of some Toeplitz operators, Proc. Amer. Math. Soc. 44 (1974), 129-134.

5. C. C. Cowen, Hyponormality of Toeplitz operators, Proc. Amer. Math. Soc. 103 (1988), 809-812.

6. C. C. Cowen and J. J. Long, Some subnormal Toeplitz operators, J. Reine Angew. Math. 351 (1984), 216-220.

7. R. G. Douglas, Banach algebra techniques in operator theory, Academic Press, New York, 1972.

8. J. Garnett, Bounded analytic functions, Academic Press, New York, 1981.

9. P. R. Halmos, Ten problems in Hilbert space, Bull. Amer. Math. Soc. 76 (1970), 887-933.

10. T. Ito and T. K. Wong, Subnormality and quasinormality of Toeplitz operators, Proc. Amer. Math. Soc. 34 (1972), 157-164.

11. T. Nakazi, Intersection of two invariant subspaces, Canad. Math. Bull. 30 (1987), 129-133. 
12. __, Absolute values of Toeplitz operators and Hankel operators, Canad. Math. Bull. 34 (1991), 249-253.

13. J. Neuwirth and D. J. Newman, Positive $H^{1 / 2}$ functions are constant, Proc. Amer. Math. Soc. 18 (1967), 958.

14. S. C. Power, Hankel operators on Hilbert space, Pitman, Boston, Mass., 1982.

Department of Mathematics, Faculty of Science, Hokkaido University, Sapporo 060, JAPAN 